\title{
Imprimindo o espaço para as pessoas com deficiência visual: uma revisão sistemática
}

\author{
Printing the environment for visually impaired users: a systematic review
}

\author{
- Gabriel Moraes de Bem \\ Universidade Federal de Santa Catarina, Brasil \\ gabrieldebem@gmail.com \\ Regiane Trevisan Pupo \\ Universidade Federal de Santa Catarina, Brasil \\ regipupo@gmail.com
}

\begin{abstract}
This paper presents the results of a systematic review of 13 databases that aimed to identify which rapid prototyping techniques (RP) have been applied to development of tactile maps and tactile models. For this purpose were adopted the key words tactile map; tactile model; tactile scale model; additive manufacturing; digital fabrication; and rapid prototyping. Only 11 results deal with the PR in the manufacture of the devices. As a result of four cases reviewed, there are benefits from psychological and learning fields and also technical such as the resolution of the elements, its legibility and quality control in manufacturing.
\end{abstract}

Keywords: Visually Impaired User, Mobility, Wayfinding, Digital Prototyping

\section{Introdução}

Na fabricação de mapas e maquetes táteis, de acordo com Edman (1992), os elementos devem ser representados com tamanha clareza que não prejudiquem a sua leitura. Relacionado a isso, estudos mostraram que a aplicação da prototipagem rápida(PR) para fins táteis possibilita a fabricação de texturas elaboradas de maneira clara, propiciando conforto tátil na leitura e durabilidade quanto à utilização por parte dos usuários na leitura dos materiais, (Celani \& Milan, 2007; Ferreira \& Ferreira, 2012; Pupo \& Aranda, 2011).

Com relação à utilização dos mapas, de acordo com Rowell e Ungar (2005), há pessoas com deficiência visual que preferem fazer uma leitura prévia do mapa na sua residência, antes de se deslocarem para o destino. Nesse sentido, a aplicação da PR na elaboração de matrizes táteis de mapas cartográficos para posterior termoformagem com película de PVC, por exemplo, obteve eficiência na reprodução dos relevos para posterior leitura pelos usuários (Ferreira \& Ferreira, 2012).

Dessa forma, visando o embasamento da pesquisa frente a uma variedade de opções e formas de se representar o espaço para um público específico, o objetivo desse estudo, se volta para uma revisão sistemática de teses, dissertações, artigos de periódicos e patentes, em 13 bases de dados online, a fim de identificar quais as técnicas de PR já foram aplicadas para elaboração de mapas e maquetes táteis e seus respectivos resultados. Assim sendo, o resultado dessa pesquisa poderá direcionar a um aprofundamento nas técnicas de maior eficiência para tal leitura. Isso, certamente, irá contribuir para a consolidação de um método a ser seguido no Brasil, tendo em vista que a norma brasileira que regulamenta aspectos relacionados à acessibilidade a edificações, mobiliário, espaços e equipamentos urbanos, NBR 9050:2004, não trata de uma série de elementos para sua fabricação. Como exemplo, podese citar: 1) tipo de informação que deve ser representada; 2) qual a linguagem empregada (braille, relevo, libras); 3) quais os tipos de usuários que esses equipamentos devem atender; 4) quais os tipos de materiais mais adequados para a sua fabricação, leitura e 5) quais as suas dimensões.

\section{Metodologia}

Para realização dessa etapa, a pesquisa partiu de uma revisão sistemática optando-se pelo recorte entre os anos de 2004 a 2014, em virtude do ano de publicação da norma brasileira NBR 9050:2004, na busca de publicações sobre o tema mapas táteis e mobilidade. A partir desses resultados, foi feita uma nova classificação para identificar quais publicações tratavam dos assuntos mapa tátil e maquete tátil, associadas à PR.

Os mapas táteis, de acordo com o Catálogo Nacional de Produtos para Tecnologia Assistiva, baseado na lista de definições da ISO 9999:2007, são classificados nos itens 12 e 22 , sendo este último relacionado a produtos de apoio para comunicação e informação e o primeiro para produtos de apoio 
para a mobilidade pessoal (Ministério da Ciência, Tecnologia e Inovação, 2015).

Cabe ainda destacar a diferença entre maquete tátil e mapa tátil, em virtude dos resultados que poderão ser encontrados, que consistem na maneira com que os elementos são reproduzidos. Na maquete tátil, há a representação real de forma volumétrica e em menor escala. Já o mapa tátil traz a informação codificada em caracteres simbólicos como pontos, linhas e superfícies (Gual et. al, 2011).

A análise empregada surgiu a partir de uma revisão bibliográfica exploratória, de um conjunto de artigos resultantes de uma busca no portal Capes. Utilizando-se as palavras chaves de interesse, "tactile map"; "tactile model"; "tactile scale model"; "additive manufacturing"; "digital fabrication"; e "rapid prototyping", identificaram-se os seguintes periódicos relacionados ao tema:

a) Transactions of Japan Society of Kansei Engineering;

b) Professional Geographer

c) The British Journal of Visual Impairment

d) Journal of Visual Impairment \& Blindness

e) Modern Physics Letters B

f) Journal of Engineering Design

A partir disso, uma nova pesquisa foi feita no mesmo portal para identificar as bases de dados que detinham as publicações de tais periódicos. Assim, dentre as bases disponíveis para pesquisa no portal, selecionou-se as seguintes bases:

a) Academic Search Complete - EBSCO; (BDTD);

b) Biblioteca digital brasileira de teses e dissertações

c) Banco de Teses - CAPES;

d) Civil Engineering Abstracts (CSA/ASCE ProQuest);

e) Compendex - Engineering Village;

f) Cumulative Index of Computer Aided Architectural Design (CUMINCAD);

g) Dissertations and Theses - ProQuest;

h) Japan Science and Technology Information Aggregathor Eletronic (J-Stage);

i) Sage Journals Online (SAGE);

j) Science Direct;

k) Scopus;

l) Web of Science;

m) Wiley Online Library

Em cada uma das bases, foi feita uma nova busca (pesquisa avançada), respeitando-se as orientações disponíveis nas ferramentas de ajuda/help de cada uma delas, a fim de refinar a pesquisa e se obter dados mais objetivos. Os critérios de inclusão de resultados foram definidos da seguinte forma: quanto ao tipo de documento, foram incluídos artigos de periódicos revisados pelos pares, teses e dissertações, conferências, monografias, anais de congressos, capítulos de livros, material de referência e patentes; com relação ao período de tempo, adotou-se o intervalo de 2004 à 2015 em virtude do período de vigência da NBR9050, que trata dos aspectos relacionados à acessibilidade do Brasil. Para o idioma do documento optou-se por inglês, português e espanhol.
Sabe-se que uma mesma referência pode estar cadastrada em mais de uma base, o que pode gerar falsas impressões em relação à exclusividade do conteúdo e o número de fontes de informação disponibilizadas pelas bases. Além disso, uma mesma base pode trazer como resultado da busca a referência de todos os artigos publicados nos anais do evento e também a referência de cada um dos artigos que faz parte dos anais, o que acaba por configurar também uma duplicidade de referências.

Após a exclusão das referências que estavam em duplicidade, os dados foram tabulados para a realização de uma série de análises em relação às publicações. Foi possível dividir o resultado das buscas em que os termos utilizados nos tópicos da pesquisa foram encontrados, em cinco grandes áreas de aplicação:

a) Ensino/aprendizagem, quando as publicações estavam relacionadas ao emprego em ambiente escolar, no aprendizado de diversas disciplinas, resultante da aplicação dos elementos táteis.

b) Cartográfico/geográfico está restrito ao aprendizado de conteúdo exclusivo das áreas cartográficas e geográficas, independente do fim ser educacional (ambiente escolar) ou não.

c) A classificação inclusão social está relacionada aos mecanismos para inclusão de pessoas com deficiência no cotidiano, como por exemplo, na percepção da arte.

d) As publicações identificadas como diversa dizem respeito às produções onde os termos de busca foram encontrados, porém não estão relacionados necessariamente a pessoas com deficiência ou ainda com mapas táteis para auxílio à mobilidade.

e) As publicações classificadas como mapa tátil/ mobilidade, dizem respeito não somente à fabricação, uso e avaliação de mapas táteis para mobilidade, mas também a aspectos relacionados com a sua fabricação e estudo. Como exemplo, critérios para elaboração de simbologias, aspectos cognitivos da leitura tátil, desempenho de equipamentos para sua fabricação, dispositivos associados ao uso desses, ou seja, todo e qualquer resultado que possa contribuir para o conhecimento do tema e enriquecimento de informações para a elaboração de um mapa tátil.

Para o presente estudo, apenas os resultados identificados no item Mapa tátil/Mobilidade foram relevantes.

\section{Resultados}

De um total de 229 resultados encontrados a partir dos critérios e palavras chave de busca adotadas, 103 foram relacionados ao tema mapa tátil/mobilidade, sendo que apenas 11 tratam da implementação da PR na fabricação dos dispositivos, sejam eles mapas ou maquetes táteis. Os demais estão voltados para estudos cognitivos, percepção do usuário, avaliação de simbologias adotadas em mapas, implementação de dispositivos sonoros e etc. Tal panorama mostra o pouco número de estudos relacionados ao tema.

As novas técnicas de prototipagem digital aditiva, tais como, Stereolitografia (SLA), Sinterização Seletiva a Laser (SLS), 
Fused Deposition Modeler (FDM), entre outras, permitem que a informação seja representada com uma maior complexidade volumétrica. Dessa forma, esse sistema de produção expande as possiblidades de melhoraria dos dispositivos táteis voltados para auxílio das pessoas com deficiência visual (Chua, Leong, Lim, 2003 apud Gual et. al, 2011).

A seguir, apresentam-se 04 estudos de caso da utilização das técnicas de prototipagem digital para fabricação de mapas e maquetes táteis resultantes da pesquisa.

\section{Estudo de caso 1}

A técnica de impressão 3D colorida foi implementada em um estudo para avaliar a utilização de mapa tátil impresso em 3D sob os princípios do Desenho Universal, desenvolvido por Gual et. al, 2011. Foi desenvolvido um mapa (Figura 1) que trouxe não somente volumetrias em 3D, mas também características típicas de mapas táteis $2 \mathrm{D}$, juntamente com a legenda. $O$ dispositivo foi desenvolvido para ser utilizado tanto com a percepção tátil quanto visual, ampliando assim a possibilidade de utilização.

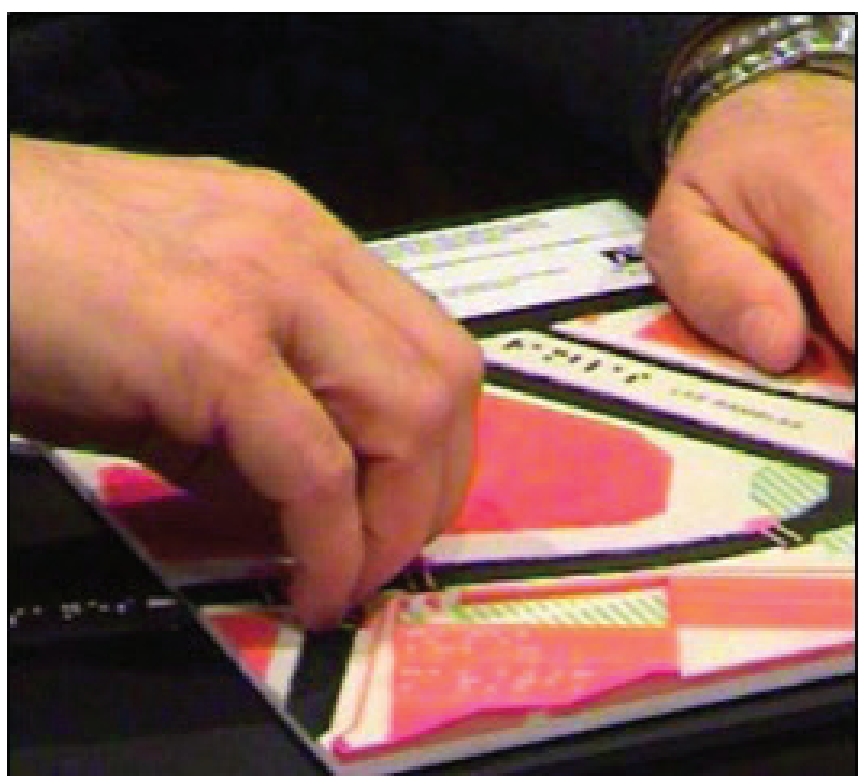

Figura 1: Mapa tátil em impressão 3D colorida. Fonte: Gual et. al. (2011).

O mapa apresenta uma área da cidade de Barcelona, Ciutat Vella, e particularmente onde está localizado o museu Mrítim. O objetivo da representação dessa área foi facilitar o acesso à cultura por parte das pessoas com deficiência visual, tendo em vista queo museu dispõedeequipamentosedispositivos voltados para a acessibilidade desses usuários. Dessa forma, os usuários poderiam aumentar a sua participação social e conhecimento histórico e cultural da região histórica da Catalônia.

\section{Estudo de caso 2}

Celani e Milan (2007) desenvolveram uma pesquisa para elaboração de maquetes táteis, que dentre os objetivos, pretendiam responder à seguinte pergunta:É possível construir maquetes táteis com a utilização da Prototipagem Digital?

Empregando-se a técnica de Sinterização Seletiva à Laser, foram produzidas duas maquetes: a primeira delas com o objetivo de orientar os usuários como acessar o laboratório de acessibilidade da biblioteca central da UNICAMP, situado no segundo pavimento do prédio, partindo do acesso principal. Esse modelo foi dividido ainda em duas partes, um deles representando o segundo andar e ou outro o primeiro. Ambas as peças apresentavam setas para direcionar o caminho. Osegundo modelo (Figura 2), apresentando o interior do laboratório, com a disposição das mesas, prateleiras e equipamentos, contudo, sem apresentar a rota definida (Celani \& Milan, 2007).

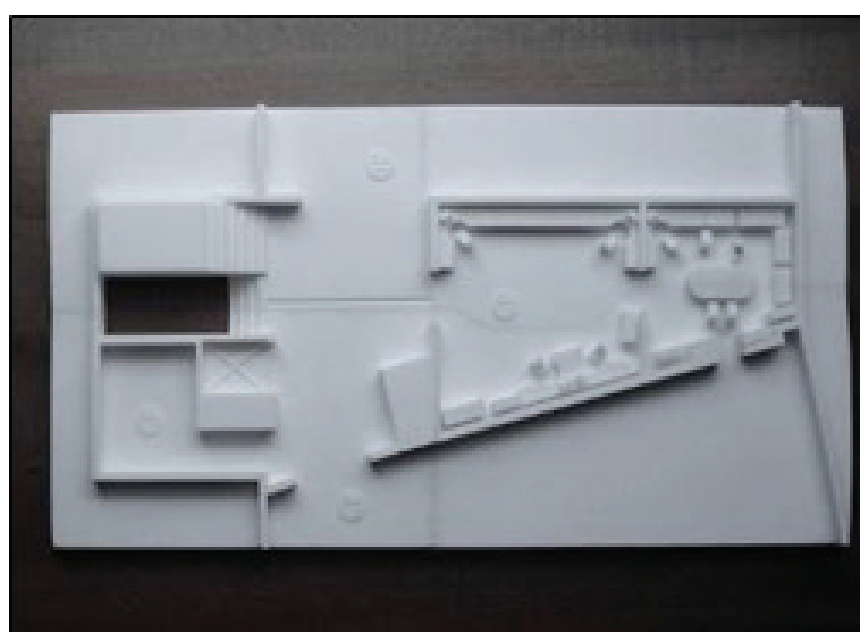

Figura 2: Mapa tátil em Sinterização Seletiva à Laser. Fonte: Celani e Milan (2007).

Os modelos fabricados para a pesquisa foram produzidos no Laboratório de Prototipagem Rápida do Centro de Pesquisas Renato Archer (CENPRA), hoje chamado de Centro de Tecnologia da Informação Renato Archer (CTI), em Campinas, Brasil, sendo posteriormente avaliados por seis voluntários, de diferentes idades e graus de deficiência visual, tanto mulheres quanto homens (Celani \& Milan, 2007).

\section{Estudo de caso 3}

Um exemplo do emprego das técnicas de prototipagem digital para elaboração de maquetes táteis, as quais podem ser empregadas para proporcionar a aproximação das pessoas com deficiência visual do contexto histórico (Voigt \& Martens, 2006), é o resultado da tese de doutorado de Christoph Oberhofer, desenvolvida na Universidade de Tecnologia de Viena, na Áustria, na reconstrução de uma sinagoga desenhada pelo arquiteto Jacob Modern e destruída em 1938 (Martens \& Stellingwerff, 2005).

Para tal reprodução, optou-se pela junção de duas técnicas de prototipagem digital: corte à laser e impressão baseada em pó de celulose, dividindo-se o elemento em peças passíveis de representação plana e volumétrica em virtude do tempo e custo de fabricação dos elementos (Figura 3) (Oberhofer, 2005). 


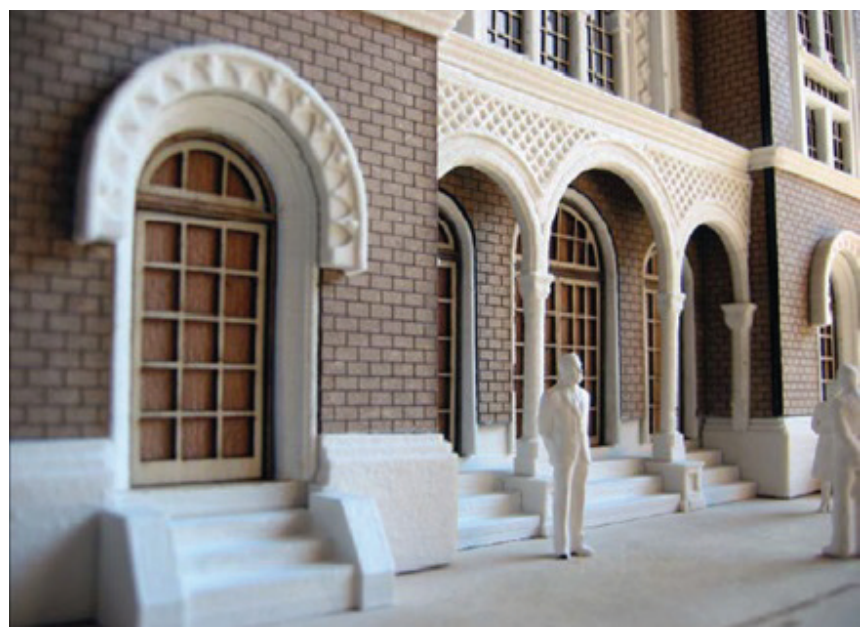

Figura 3: Maquete tátil em corte à laser e impressão 3D. Fonte: Oberhofer (2005).

\section{Estudo de caso 4}

Outro exemploda combinação de duas técnicas de Prototipagem Digital é o trabalho desenvolvido por Capeli e Bernardi, durante os anos de 2010 e 2011. Em trabalho de Iniciação Científica cujo objetivo era o aprimoramento de um mapa tátil com recursos sonoros para a área central do Campus da UNICAMP, com o desenvolvimento de simbologias e instrumental apropriado para a leitura e manipulação por pessoas com baixa visão e cegueira, além de verificar a sua usabilidade e incluir recursos tecnológicos que possibilitassem a obtenção da informação de forma fácil e correta (Capeli \& Bernardi, 2011).

$O$ primeiro processo utilizado foi o de corte à laser em placas de laminado, para representação da rota que os usuários deveriam seguir quando do deslocamento naquela região do Campus. A segunda técnica, impressão 3D baseada em pó (Figura 4), foi utilizada para a fabricação das legendas em braile e das edificações existentes no Campus.

Após a elaboração dos modelos, os mesmos foram submetidos à analise pelos usuários para verificação e ajustes dos protótipos, determinação da melhor escala de representação das simbologias adotadas e dos materiais e texturas empregados.

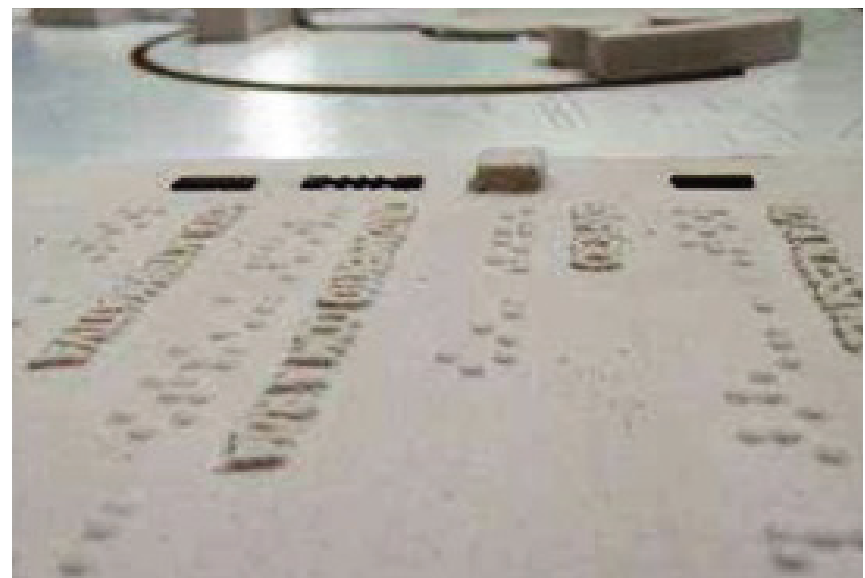

Figura 4: Legenda e edificações existentes em impressão 3D. Fonte: Capeli e Bernardi (2011).
Como resultado dos estudos de casos analisados, em relação aos aspectos psicológicos e de aprendizagem da utilização de mapas e maquetes prototipados percebeu-se que: (1) as formas volumétricas do desenho aumentam as possibilidades relacionadas à interpretação tátil, diferentemente dos mapas fabricados com os métodos convencionais; (2) a utilização do mapa impresso favoreceu a elaboração do mapa cognitivo, que, de acordo com Bins et al (2002), corresponde ao modo como são organizadas mentalmente as informações ambientais percebidas pelos usuários.

Já quanto aos aspectos técnicos relacionam-se: (1) a resolução do produto permitiu uma reprodução perfeita das legendas em braile, com consequente facilidade de leitura; (2) o modelo, além de leve, apresentou resistência suficiente para ser manipulado pelos usuários durante a leitura tátil; (3) a associação de diferentes técnicas de fabricação de um mesmo produto podem minimizar os custos da produção; (4) os parâmetros de controle de fabricação de mapas impressos são superiores e relevantes no resultado, se comparados aos processos usuais de fabricação como termoformagem e papel micro-encapsulado.

\section{Discussão}

Mapas e maquetes táteis são de grande relevância no auxílio à mobilidade das pessoas com deficiência visual (Voigt \& Martens, 2006). A possibilidade de representar a arquitetura tanto da edificação quanto dos espaços e a relação entre seus elementos, com maior precisão e clareza dos detalhes, facilita o processo de leitura e entendimento por parte dos seus usuários.

A prototipagem rápida surge então como uma ferramenta extremamente útil na confecção dos modelos táteis. Uma das características que pode ser destacada é a possibilidade de representação com grande resolução das texturas, símbolos e texto em braile facilitando a sua leitura, além da resistência do produto à dinâmica associada a sua leitura.

Além disso, ainda que em cada um dos exemplos citados da utilização da prototipagem rápida na fabricação dos mapas táteis apenas um modelo tenha sido produzido, a tecnologia de PR permite que cópias sejam geradas mais rapidamente, tendo-se disponível os arquivos necessários, o que permite também o cálculo de custo tanto de material quanto de equipamentos (Voigt \& Martens, 2006), contribuindo assim com a disseminação do mapa ou da maquete desenvolvida em diferentes regiões.

O resultado dessa pesquisa, certamente irá contribuir para o direcionamento e ao aprofundamento quanto ao processo de fabricação a ser adotado na elaboração dos mapas e maquetes táteis, tendo em vista os resultados obtidos pelos autores resultantes da pesquisa.

\section{References}

Edman, P. (1992). Tactile graphics. American Foundation for the Blind. New York.

Bins Ely, Vera H. M., Dischinger, Marta \& Mattos, Melissa 
L. (2002). Sistemas de Informação ambiental - elementos indispensáveis para a acessibilidade e orientabilidade. Anais do VII Congresso Latino- Americano de Ergonomia, XII Congresso Brasileiro de Ergonomia, I Seminário Brasileiro de Acessibilidade Integral, Recife.

Capelli, Giovanni Andreas \& Bernardi, Núbia. (2011). Projeto de mapa tátil para área central do campus da Unicamp: desenvolvimento de recursos que auxiliem na orientação espacial de usuários com deficiência visual. Unicamp: Campinas. Relatório de iniciação científica.

Celani, G.; Milan, L. F. (2007). Tactile scale models: threedimensional info-graphics for space orientation of the blind and visually impaired. 3rd International Conference on Advanced Research in Virtual and Rapid Prototyping. Sep 24-29, Leiria, Portugal.

Ferreira, M. E. S. \& Ferreira, L. F. (2012). Construção de matrizes táteis pelo processo de prototipagem rápida. Revista Brasileira de Cartografia, v. 64, p.45-55.

Gual, J., Puyuelo, M. \& Lloveras, J. (2011). Universal design and visual impairment: tactile products for heritage acess. International Conference on Engineering Design, ICED 2011.
Technical University of Denmark. Spain.

Martens, B. \& Stellingwerff, M. (2005). Creating Physical Models Using Virtual Reconstructions: Mixed CAM techniques for a Viennese synagogue scale model. In: Angulo, A. \& Vasquez, V. G. Proceedings of the 9th Iberoamerican Congress of Digital Graphics - SIGraDi, Lima, vol. 1, pp. 108-113.

Ministério da Ciência, Tecnologia e Inovação (2015) Catálogo nacional de produtos de tecnologia assistiva". Retrieved from http://assistiva.mct.gov.br/.

Oberhofer, C. (2005). Computergestützte Rekonstruktion der Synagoge in Wien Währing. 2005. 125 f. Thesis. Vienna University of Technology.

Pupo, R. T. \& Aranda, I.M. (2011). Sentir um quadro. In: Congresso de La Sociedad Iberoamiricana de Gáfica Digital (SIGraDi) 15. Santa Fé, Argentina.

Rowell, J. \& Ungar, S. (2005). Feeling our way: tactile map user requirements - a survey. Proceedings of XXII International Cartographic Conference, La Coruna.

Voigt, A. \& Martens, B. (2006). Development of 3D tactile models for the partially sighted to facilitate spatial orientation. 24th eCAADe Conference Proceedings, Volos, Greece, 366-370. 